\title{
1 Genomic basis of transcriptome dynamics in rice under field conditions
}

2

3 Makoto Kashima ${ }^{1}$, Ryota L. Sakamoto ${ }^{2}$, Hiroki Saito ${ }^{3,4}$, Satoshi Ohkubo ${ }^{3,5}$, Ayumi

4 Tezuka $^{1}$, Ayumi Deguchi ${ }^{1}$, Yoichi Hashida ${ }^{1}$, Yuko Kurita ${ }^{1}$, Koji Iwayama $^{5}$, Shunsuke

$5 \quad$ Adachi $^{5}$ and Atsushi J. Nagano ${ }^{7 *}$

6

$7 \quad{ }^{1}$ Research Institute for Food and Agriculture, Ryukoku University, Shiga, Japan

$8 \quad{ }^{2}$ Seibi Senior High School, Gifu, Japan

$9 \quad{ }^{3}$ Graduate School of Agriculture, Kyoto University, Kyoto, Japan

$10{ }^{4}$ Tropical Agriculture Research Front, Japan International Research Center for

11 Agricultural Sciences, Okinawa, Japan

$12{ }^{5}$ Faculty of Data Science, Shiga University, Shiga, Japan

$13{ }^{6}$ Institute of Global Innovation Research, Tokyo University of Agriculture and

14 Technology, Fuchu, Tokyo, Japan

$15{ }^{7}$ Faculty of Agriculture, Ryukoku University, Shiga, Japan

16

17 
18 How genetic variations affect gene expression dynamics of field-grown plants remains unclear. Using statistical analysis of large-scale time-series RNA-sequencing

20 of field-grown rice from chromosome segment substitution lines (CSSLs), we identified 1675 expression dynamics quantitative trait loci (edQTLs) leading to polymorphisms in expression dynamics under field conditions. Based on the edQTL and environmental information, we successfully predicted gene expression under environments different from training environments, and in rice cultivars with more complex genotypes than the CSSLs. Overall, edQTL' identification helped understanding the genetic architecture of expression dynamics under field conditions, which is difficult to assess with laboratory experiments ${ }^{1}$.The prediction of expression based on edQTL and environmental information will contribute to crop breeding by increasing the accuracy of trait prediction under diverse conditions. genetic backgrounds, developmental stages, and physiological status. This variability can cause missing heritability in crop breeding and low accuracy in medicine ${ }^{2-4}$.

33 Environmental stimuli can induce transcriptional responses directly and/or indirectly. Measuring transcriptome dynamics is a comprehensive method for assessing 
physiological factors. The expression quantitative trait loci (eQTL) approach is frequently

used to assess the association between genetic variation and gene expression

polymorphism $^{5-9}$. Because this approach requires transcriptome data for the complete set

of a QTL mapping population under the given conditions ${ }^{5-9}$, only a limited range of

environmental conditions are covered. Contrarily, statistical models based on

meteorological information, circadian clock, and developmental age have succeeded in

describing transcriptome dynamics in the field ${ }^{10-12}$, although these models can only

predict the transcriptome of one or few genotypes used as training data ${ }^{10-12}$. Thus, no

study has clarified the relationship between genetic variation and transcriptome dynamics

under fluctuating field conditions.

Here, we first defined the loci that determine polymorphisms in gene expression

dynamics as 'edQTL'. edQTL can explain polymorphisms in gene expression dynamics under a broad range of environmental conditions, while eQTL can only explain them under the investigated environment (Fig. 1a). Our approach consists of four steps: timeseries RNA-sequencing (RNA-Seq), prediction model development, edQTL detection,

51 and evaluation of edQTL (See Methods, Fig. 1b, and Supplementary Fig. 1). We used the rice (Oryza sativa L.) cultivars 'Koshihikari' (a leading japonica cultivar) and 'Takanari'

53 (a high-yield indica cultivar) ${ }^{13,14}$ that would present substantial polymorphisms in 
expression dynamics. The 78 reciprocal CSSLs and two backcross inbred lines (BILs)

2a). To refelct the effect of plant age (days after seeding) in our model, we prepared four bihourly sampling for $24 \mathrm{~h}$ were conducted from May to September 2015 (cropping season in Japan). At each sampling time, the youngest fully expanded leaves were collected from two different genotypes at each transplant set while withered plants were excluded from the sampling, which could not be sampled (Fig. 1c, Supplementary Fig. 2b, and Table S2). The 926 individual leaves were applied for RNA-Seq, to obtain transcriptome data. After filtering samples and genes (Supplementary Fig. 3a, b) and confirming genotypes (Supplementary Fig. 3c and 4), 23,924 expressed genes from 854 samples were used. Correlation analysis of the transcriptome data showed an obvious transcriptome-wide diurnal variation in the expressed genes (Fig. 1d).

We then developed two prediction models describing transcriptome dynamics under fluctuating field environments in 'Koshihikari' and 'Takanari'. In this step, we used the statistical modeling tool ' $\mathrm{FIT}^{\prime 10}$ for predicting transcriptome dynamics under field conditions. As most of the genome in the CSSLs was not substituted (Supplementary Fig 2a), the expression dynamics of most genes was expected to be identical to that of the 
background parent. Thus, the RNA-Seq data of 'Koshihikari'- and 'Takanari'-background

samples were used to develop the prediction model for 'Koshihikari' and 'Takanari' lines,

respectively. Circadian clock and meteorological data (air temperature and global solar

radiation) (Supplementary Fig. 5a) were considered in model development and "scaled

age" was used to adjust differences in heading date among rice genotypes (See Methods

and Supplementary Fig. 5). We found polymorphisms in the predicted expression

dynamics of 3696 genes (15.4\% of the expressed genes; Supplementary Fig. 3e and Table

S3). For instance, the 'Koshihikari' model for Os09g0343200 showed an obvious diurnal

oscillation in expression, whereas the 'Takanari'-model showed a constant low-level

expression (Fig. 2a). Notably, in some 'Takanari'-background CSSLs, SL1329 and

SL1330, the expression of Os09g0343200 resembled that of the 'Koshihikari' model

rather than the 'Takanari'-model (Fig. 2a), suggesting that genetic substitution in the

chromosome 9 affected $O s 09 g 0343200$ expression.

The CSSLs had been previously genotyped using 141 simple sequence repeat (SSR) markers (Supplementary Fig. 2a) ${ }^{14}$. As so, at the edQTL detection step, we searched for the SSR markers explaining the expression dynamics polymorphisms. We evaluated the decrease in the residual error of each gene assuming edQTL around each SSR marker 
90 significantly decreased only by assuming edQTL on chromosome 9 (Fig. 2b and

91 Supplementary Fig. 6b), indicating that this is a cis-edQTL. The prediction models chosen

92 for each sample based on edQTL genotypes explained the expression dynamics of

93 Os09g0343200 better than their background genotypes (Fig. 2c). Overall, edQTL were

94 identified for 1675 genes $(45.3 \%$ of genes with expression dynamics polymorphism between 'Koshihikari' and 'Takanari', false discovery rate $=0.05$; Supplementary Fig. 6f), including 222 genes affected by trans-edQTL (Fig. 2d). Forty-three genes were

97 affected by multiple edQTL. For 33 of these genes, the sum of residual errors based on all edQTL was smaller than that of the most significant edQTL alone (Supplementary Fig. 7). Detailed results for individual genes can be found in our database

100 (https://ps.agr.ryukoku.ac.jp/osa_edqtl). A cis-edQTL could explain the expression dynamics polymorphism of $O s 01 g 0537250$ that be specifically observed in young plants (Fig. 2e,f). The expression depended on time of day in both parental models, but scaled age was only important in the 'Koshihikari' model (Fig. 2e,f and Supplementary Fig. 8).

104 The edQTL-affected 393 genes depending on the time of day and 275 genes depending on scaled age (Supplementary Fig. 9). 
108 environments from where training data were obtained for model development.

109 'Koshihikari', 'Takanari', and the CSSLs of two transplant sets were cultivated in a field

110 different from that used in 2015 and sampled in August 2016; 139 RNA-Seq datasets were

111 obtained (Supplementary Fig. 2c and Table S2). Environmental factors differed between

112 the 2015 and 2016 fields (Supplementary Fig. 10a). For $91.8 \%$ of the 1675 genes affected

113 by the edQTL, the prediction was improved by the edQTL model compared with the BG

114 model (Fig. 3a,b and Supplementary Fig. 10b). Furthermore, the prediction of the

115 expressed genes for the validation dataset showed comparable accuracy to that of the

116 training dataset (Supplementary Fig. 11). Thus, the identified edQTL could explain the

117 expression dynamics polymorphisms in different years and locations. For further

118 verification, two BILs between 'Koshihikari' and 'Takanari' (HP-a and HP-b) ${ }^{15,16}$ were

119 cultivated at Takatsuki in 2015, and five and six RNA-Seq datasets were obtained for HP-

$120 \mathrm{a}$ and HP-b, respectively (Supplementary Fig 2b and Table S2). These lines carried

121 'Koshihikari' alleles at $16.3 \%$ and $19.9 \%$ of their markers with the genetic background of

122 'Takanari' (Supplementary Fig 2a). To evaluate the performance of the edQTL-based

123 prediction, we calculated the sum of prediction errors of all edQTL-influenced genes

124 using edQTL-, 'Koshihikari'-, and 'Takanari'-models. Overall, the edQTL model 
126 b genomes showed the significant advantage of the edQTL model $(p<0.001)$ (Fig. $3 c, d)$.

127 For instance, as the genotype of the trans-edQTL for Os03g0388300 comprised the

128 'Koshihikari' allele in the genome of HP-a and the 'Takanari' allele in the genome of HP-

129 b (Fig. 3e), 'Koshihikari'- and 'Takanari'-models were used to predict Os03g0388300

130 expression in the edQTL models for HP-a and HP-b, respectively. The expression of

131 Os03g0388300 fluctuated with time and it was higher in 'Koshihikari' than in 'Takanari'

132 (Supplementary Fig. 12a). The prediction of Os03g0388300 expression based on the

133 edQTL models was better than the prediction based on the BG models (Fig. 3f).

134 Regarding OsKS3 (Os04g0611700) ${ }^{17}$ in HP-b, the prediction based on the edQTL model

135 was worse than that based on the BG model (Supplementary Fig. 12b,c). True edQTL

136 might therefore exist around SSR markers 52 or 53, which were not substituted with the

137 'Koshihikari' allele in HP-b (Supplementary Fig. 12b). Such difficulty may occur in some

138 edQTL-based predictions for the BILs, because several edQTLs identified by the CSSLs

139 can be unlinked to the substituted genome regions in the BILs. Finally, we concluded that

140 our approach successfully identified loci linked to expression dynamics polymorphisms

141 in field conditions.

142 The edQTL approach scans a broader range of conditions (Fig. 1a) but it is less

143 sensitive when focusing on a specific condition. This is because eQTL are expected to 
144 show the same effects on gene expressions in all samples used in a study. Contrarily, some

145 edQTL were expected to affect gene expressions in some specific samples, depending on

146 their environments. The lower sensitivity of the edQTL approach relative to that of the

147 eQTL approach might explain the smaller fraction of trans-edQTL compared with that of

148 trans-eQTL in previous studies $\left(13.3 \%\right.$ and $62-71 \%{ }^{6,8,9}$, respectively) because trans-

149 edQTL/eQTL have generally smaller effect sizes than cis-edQTL/eQTL $^{6,8}$.

150 Phenotypic plasticity plays key roles in plant's environmental adaptation ${ }^{18,19}$.

151 Nevertheless, as this is difficult to study in natural environments, the genetic architecture

152 of phenotypic plasticity under field conditions remains largely unveiled. The edQTL

153 identified by our novel method improve the understanding of the genetic architecture

154 underlying the expression dynamics polymorphisms between 'Koshihikari' and 'Takanari'

155 in the field. In addition, our method showed that rice gene expression dynamics can be

156 predicted based on genotypic and meteorological information. In crop breeding,

157 polymorphisms of environmental responses result in unexpected performance of a bred

158 crop under environments differing from experimental fields. Because the transcriptome

159 can be beneficial for trait $\operatorname{prediction}^{20-23}$ and our approach allows transcriptome

160 predictions under various conditions, it contributes for crop breeding and for

161 understanding plant systems. 
bioRxiv preprint doi: https://doi.org/10.1101/451609; this version posted January 31, 2020. The copyright holder for this preprint (which was not certified by peer review) is the author/funder. All rights reserved. No reuse allowed without permission.

162 
164 Overview of edQTL identification and verification. Our approach consisted of four

165 steps (Fig. 1b). First, the parent rice lines and their descendants were cultivated in a paddy

166 field and sparsely sampled at several time points for RNA-Seq (Time-series RNA-Seq,

167 Fig. 1b and Supplementary Fig. 1). Second, parental prediction models were developed to describe environmental responses in 'Koshihikari' and 'Takanari' in terms of gene expression (Prediction model development, Fig. 1b). Most gene expression dynamics would be identical among the CSSLs and the parent with the same background genotype.

171 Thus, we used 'Koshihikari'- and 'Takanari'-background CSSLs as well as their parents to develop the parental models (Fig. 1b and Supplementary Fig. 1). Third, the dependency of expression dynamics polymorphisms on genetic variation was statistically evaluated based on comparisons between predictive gene expression and observed gene expression in CSSLs assuming that genetic variation of SSR markers leads to expression dynamics polymorphisms (Supplementary Fig. 6) (edQTL detection, Fig. 1b). Fourth, by integrating

177 the parental models and edQTL information, gene expression dynamics was predicted based on environmental and genotypic information (Evaluation of edQTL).

Plant materials. We cultivated the following rice (O. sativa) lines: the japonica variety 
181 background lines, except for SL1213, and 38 'Takanari'-background lines, except for

182 SL1306) ${ }^{15}$, and two 'Takanari'-background BILs (HP-a and HP-b) ${ }^{15,16}$. Each variety and

183 line were sown in nursery trays. Approximately one month after sowing, seedlings were

184 transplanted to a paddy field at Takatsuki, Japan $\left(34^{\circ} 51^{\prime} 19^{\prime \prime} \mathrm{N}, 135^{\circ} 37^{\prime} 51^{\prime \prime} \mathrm{E}\right)$ in 2015 and

185 at Kizugawa, Japan $\left(34^{\circ} 4^{\prime} 05^{\prime \prime} \mathrm{N}, 135^{\circ} 50^{\prime} 33^{\prime \prime} \mathrm{E}\right)$ in 2016 . To consider the effect of plant age

186 in the prediction of gene expression dynamics, four and two transplant sets were prepared

187 in 2015 and 2016, respectively (Fig. 1c and Supplementary Fig. 2b,c). Seed sowing and

188 transplanting were conducted according to the following schedules. In 2015, transplant

189 set 1: Seed sowing date "2015-04-03", Transplanting date "2015-05-01"; transplant set 2:

190 Seed sowing date "2015-04-17", Transplanting date "2015-05-08"; transplant set 3: Seed

191 sowing date "2015-05-01", Transplanting date "2015-05-22"; transplant set 4: Seed sowing date "2015-05-15", Transplanting date "2015-06-05". In 2016, transplant set 1:

193 Seed sowing date "2016-04-21", Transplanting date "2016-05-16"; transplant set 2: Seed sowing date "2016-05-19", Transplanting date "2016-06-08".

Sampling and RNA extraction. Sixteen sets (2015) and three sets (2016) of bihourly sampling for $22 \mathrm{~h}$, from 16:00 on one day to 14:00 on the next, were conducted on the 
199 August, 24-25 August, 31 August to 1 September, 7-8 September, 14-15 September, 21-

201 We applied a stratified randomization strategy to the sampling schedule to avoid biased

202 sampling of each line over seasons. We separated individual plants into four and two

203 groups in each transplant set in 2015 and 2016, respectively, each containing the same

204 number of individuals per line. Then, the order of sampling was randomized in each group.

205 According to the sampling schedule, two plants from the 82 genotypes of each transplant

206 set were sampled. Because aged rice withered, several samples were missed at the latest

207 cultivation (Supplementary Fig. 2b). Transplant set and sampling time for each sample

208 are listed in Table S2. The youngest fully expanded leaf from each plant was collected,

209 immediately frozen in liquid nitrogen, and stored at $-80^{\circ} \mathrm{C}$ until RNA isolation for RNA-

210 Seq. Individual plants were only sampled once to avoid wounding response. Thus, all

211 RNA-Seq data were obtained from independent plants. Frozen samples were

212 homogenized with TissueLyser II (Qiagen, Hilden, Germany), and total RNA was then

213 extracted using the Maxwell 16 LEV Plant RNA Kit (Promega, Madison, WI, USA) and

214 Maxwell 16 Automated Purification System (Promega). The concentration of RNA was

215 measured using the Quant-iT RNA Assay Kit, broad range (Thermo Fisher Scientific, 216 Waltham, MA, USA). 
217 RNA-Seq library preparation and sequencing. An automated liquid handling system

218 Freedom EVO 150 (TECAN, Zurich, Switzerland) and a thermal cycler ODTC 384

219 (INHECO, Martinsried, Germany) were utilized to prepare the RNA-Seq library of 384

220 samples simultaneously. The protocol of RNA-Seq library preparation is described below.

221 We enzymatically degraded abundant RNAs such as rRNAs in the leaves, which cause

222 wasteful consumption of sequence reads (Supplementary Fig. 13). Aliquots of 252 and

223192 types of $100-\mu$ M 60-mer antisense DNAs (IDT, San Jose, CA, USA) covering rRNAs

224 and the top eight most frequently-detected transcripts (Osp1g00110.1, Osp1g00180.1,

225 Osp1g00420.1, Osp1g00170.1, Osp1g00340.1, Osp1g00330.1, Osp1g00600.1, and

226 Os11t0707000-02) in the RNA-Seq dataset of $O$. sativa leaf were mixed in equal amounts

227 (SDRNA oligo pool) ${ }^{24,25}$. One microgram of total RNA, $1.5 \mu \mathrm{L}$ of the SDRNA oligo pool,

$2283 \mu \mathrm{L}$ of $5 \times$ hybridization buffer $(0.5 \mathrm{M}$ Tris- $\mathrm{HCl}(\mathrm{pH} 7.4)$ and $1 \mathrm{M} \mathrm{NaCl})$, and RNase-

229 free water were mixed to a total volume of $10 \mu \mathrm{L}$. The mixture was incubated to anneal

230 the antisense DNAs with the target transcripts using the thermal cycler ODTC 384 with

231 the following program: $95{ }^{\circ} \mathrm{C}$ for $2 \mathrm{~min}$, slow cooling to $45^{\circ} \mathrm{C}\left(0.1{ }^{\circ} \mathrm{C} / \mathrm{s}\right)$, and $45^{\circ} \mathrm{C}$ for

2325 min. Subsequently, $1 \mu \mathrm{L}$ of Tli Thermostable RNase H (TaKaRa, Kusatsu, Japan), $2 \mu \mathrm{L}$

233 of $10 \times$ RNase $\mathrm{H}$ digestion buffer [500 mM Tris- $\mathrm{HCl}(\mathrm{pH} 7.4), 1 \mathrm{M} \mathrm{NaCl}$, and $200 \mathrm{mM}$

$234 \mathrm{MgCl}]$, and $7 \mu \mathrm{L}$ of RNase-free water were added and mixed well. This mixture was 
235 incubated at $45{ }^{\circ} \mathrm{C}$ for 30 min to selectively digest the RNA of the DNA/RNA hybrid

236 double strand. Then, $24 \mu \mathrm{L}$ of AMPure XP (Beckman Coulter, Brea, CA, USA) beads

237 was added and the mixture was purified in a 384-well magnetic plate following the

238 manufacturer's instructions. The RNA was then eluted with $14 \mu \mathrm{L}$ of RNase-free water.

239 Ten microliters of the RNA solution, $1 \mu \mathrm{L}$ of DNaseI ( 5 unit $/ \mu \mathrm{L}), 1 \mu \mathrm{L}$ of $10 \times$ DNaseI

240 buffer (both Promega), $1 \mu \mathrm{L}$ of $100 \mathrm{mM}$ DTT (Invitrogen, Carlsbad, CA, USA), and $7 \mu \mathrm{L}$

241 of RNase-free water were mixed well. This mixture was incubated at $37^{\circ} \mathrm{C}$ for 30 min to

242 degrade the SDRNA oligo pool. Subsequently, $24 \mu \mathrm{L}$ of AMPure XP beads was added

243 and purified according to the manufacturer's instructions. The RNA was eluted with 14

$244 \mu \mathrm{L}$ of RNase-free water. Five microliters of the purified mRNA obtained was mixed with

$2454 \mu \mathrm{L}$ of $5 \times \mathrm{SS}$ buffer (Invitrogen), and $1 \mu \mathrm{L}$ of $100 \mathrm{mM}$ DTT. Fragmentation of mRNA

246 was carried out at $94{ }^{\circ} \mathrm{C}$ for $4.5 \mathrm{~min}$ and immediately cooled on ice. Subsequently, 0.6

$247 \mu \mathrm{L}$ of $100 \mu \mathrm{M}$ random primer $(\mathrm{N})_{6}$ (Promega) and $0.9 \mu \mathrm{L}$ of RNase-free water were added

248 to the mixture, which was incubated at $50{ }^{\circ} \mathrm{C}$ for $5 \mathrm{~min}$ and immediately chilled on ice to

249 relax the secondary structure of the RNA. The fragmented RNA with random hexamers

and the reverse transcription master mix $[1 \mu \mathrm{L}$ of $100 \mathrm{mM}$ DTT, $0.4 \mu \mathrm{L}$ of dNTPs $(25$ 
253 mixed. For the reverse transcription step, the mixture was incubated at $25{ }^{\circ} \mathrm{C}$ for $10 \mathrm{~min}$,

254 followed by $50 \mathrm{~min}$ at $50{ }^{\circ} \mathrm{C}$. SuperScript IV was inactivated by heating the mixture at

$25575^{\circ} \mathrm{C}$ for $15 \mathrm{~min}$. Subsequently, $24 \mu \mathrm{L}$ of AMPure XP and $12 \mu \mathrm{L}$ of $99.5 \%$ ethanol were

256 added, and the mixture was purified according to the manufacturer's protocol. The reverse

257 transcription product was eluted with $14 \mu \mathrm{L}$ of RNase-free water. The purified DNA/RNA

258 hybrid solution without beads and the second-strand synthesis master mix $[2 \mu \mathrm{L}$ of $10 \times$

259 Blue Buffer (Enzymatics, Beverly, MA, USA), $1 \mu \mathrm{L}$ of dUTP/NTP mix (Fermentas,

260 Burlington, Canada), $0.5 \mu \mathrm{L}$ of $100 \mathrm{mM}$ DTT, $0.5 \mu \mathrm{L}$ of RNase H (Enzymatics), $1 \mu \mathrm{L}$ of

261 DNA polymerase I (Enzymatics), and $5 \mu \mathrm{L}$ of RNase-free water] were mixed. This

RNase-free water. Five microliters of the dsDNA solution was used in the following step.

kit (KAPA BIOSYSTEMS, Wilmington, MA, USA) with 1/10× volume of the solutions 
271 AMPure XP. The size-selected ligation product was eluted with $15 \mu \mathrm{L}$ of $10 \mathrm{mM}$ Tris-

$272 \mathrm{HCl}(\mathrm{pH}$ 8.0). One microliter of uracil DNA glycosylase (UDG; Enzymatics) was added

273 to the size-selected ligation product. The mixture was incubated at $37{ }^{\circ} \mathrm{C}$ for $30 \mathrm{~min}$ to

274 exclude the second-strand DNA. For library amplification, $2 \mu \mathrm{L}$ of the UDG-digested

275 DNA, $1 \mu \mathrm{L}$ of $2.5 \mu \mathrm{M}$ index primer $^{24}, 1 \mu \mathrm{L}$ of $10 \mu \mathrm{M}$ universal primer ${ }^{24}, 0.5 \mu \mathrm{L}$ of RNase-

276 free water, and $5 \mu \mathrm{L}$ of KAPA HiFi HotStart ReadyMix (2×) (KAPA BIOSYSTEMS)

277 were mixed. The DNA fragments with the adapters and an index sequence were amplified

278 using a thermal cycler with the following program: denaturation at $94{ }^{\circ} \mathrm{C}$ for 2 min, 16

279 cycles at $98{ }^{\circ} \mathrm{C}$ for $10 \mathrm{~s}, 65^{\circ} \mathrm{C}$ for $30 \mathrm{~s}$, and $72{ }^{\circ} \mathrm{C}$ for $30 \mathrm{~s}$ for amplification, and $72{ }^{\circ} \mathrm{C}$

280 for $5 \mathrm{~min}$ for the final extension. Two rounds of size selection were then performed to

281 remove adapter dimers with equal volume of AMPure XP to the library solution. The

purified library was eluted with $10 \mu \mathrm{L}$ of RNase-free water. The concentration of each

library was measured by qPCR using the KAPA SYBR FAST qPCR Master Mix (2x) Kit

(KAPA BIOSYSTEMS) and the LightCycler 480 II (Roche Diagnostics, Basel,

Switzerland) to mix equal amounts of libraries. One microliter of the mixed library was

used for electrophoresis with the Bioanalyzer 2100 and the Agilent High Sensitivity DNA 
290 Calculation and normalization of RNA-Seq count data. All obtained reads were

291 processed with Trimmomatic (version 0.33$)^{26}$ using the following parameters: trailing low quality $(\mathrm{Q}<19)$ or $\mathrm{N}$ bases (LEADING:19 TRAILING:19). It also trimmed reference sequences of IRGSP-1.0_transcript ${ }^{27,28}$, rRNAs, and transcripts coded in the (version 1.3.0) $)^{29}$ and Bowtie (version 1.1.2) ( $^{30}$ with default parameters. Expected counts of each gene in the RSEM outputs were used in R (version 3.4.2) ${ }^{31}$ for the following analysis. According to a previous study ${ }^{32}$, we removed the effects of misassigned reads in multiplex sequencing experiments with HiSeq 2500 as:

$$
c_{i, m, n}=\left\{\begin{array}{r}
\tilde{c}_{i, m, n}-0.0005 \times C_{i, n}\left(\tilde{c}_{i, m, n}>0.0005 \times C_{i, n}\right), \\
0\left(\tilde{c}_{i, m, n} \leq 0.0005 \times C_{i, n}\right),
\end{array}\right.
$$

where $c_{i, m, n}$ and $\tilde{c}_{i, m, n}$ denote corrected and raw read counts of gene $i$ in sample $m$ in 
307 RNA-Seq library pool $n$, respectively. $C_{i, n}$ denotes the sum of read counts of gene $i$ in

RNA-Seq library pool $n$.

311 the antisense-oligo targets (reads per million: rpm):

$$
\begin{gathered}
r_{i, m}=\frac{c_{i, m}}{C_{m}} \times 10^{6}, \\
y_{i, m}=\log _{2}\left(r_{i, m}+0.1\right),
\end{gathered}
$$

314 where, $c_{i, m}, r_{i, m}$, and $y_{i, m}$ denote merged corrected read counts, normalized read counts, and the $\log 2$-transformed value of read counts of gene $i$ in sample $m$, respectively. $C_{m}$ denotes total read counts of transcripts except for the antisense-oligo targets in sample $m$.

RNA-Seq transcriptome datasets, respectively, with more than $10^{5}$ total read counts for all genes, except for the targets of selective depletion (Supplementary Fig. 3a). We then 
325 (DRR018360) genomes were trimmed with Trimmomatic (version 0.33$)^{26}$ using the

326 parameters stated in the previous section, except for TruSeq3-PE.fa for adapter trimming.

327 The reads were then mapped to the reference genome (IRGSP-1.0, plastid: NC_001320.1,

328 mitochondrion: NC_011033.1) ${ }^{27}$ using Bowtie2 (version 2.2.9) with option -N1, which

329 allows a mismatch in seed alignment ${ }^{33}$. By using SAMtools (version 1.3.1) ${ }^{34}$, the reads

330 mapped at multiple loci and the duplicated reads, except for each representative read,

331 were discarded. Picard (version 2.1.0) (https://broadinstitute.github.io/picard/) was used

332 to add read group information to each SAM file. All SAM files of each sample were

333 merged and a VCF file was generated using SAMtools (version 1.3.1) ${ }^{34}$ and BCFtools

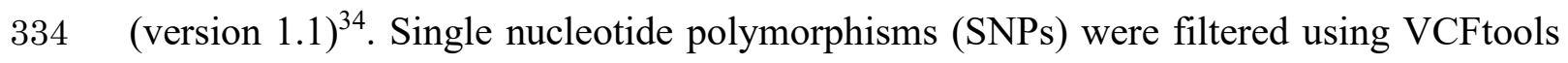

335 (version 0.1 .14$)^{35}$ with option --minDP5, which indicates that all alleles supported by

336 more than four reads are reported. The VCF file generated was analyzed using R (version

337 3.4.2) ${ }^{31}$ and the R package "vcfR" (version 1.5.0) ${ }^{36}$. We selected 15,117 homozygous

338 SNPs distinguishing 'Koshihikari' and 'Takanari' genotypes for the following analysis of

339 genotype confirmation.

340 Genotype confirmation. In previous studies, the genotypes of the CSSLs and BILs were

341 examined based on the genotypes provided by 141 SSR markers ${ }^{14}$. To confirm the 
343 obtained by RNA-Seq. First, to type 'Koshihikari'- or 'Takanari'-type SNPs in each

344 RNA-Seq sample, the trimmed RNA-Seq reads of each sample were mapped to the

345 reference genome as described in the previous section using Bowtie2 (version 2.2.9).

346 Then, the SNPs were filtered using VCFtools (version 0.1 .14$)^{35}$ with option --minDP3,

347 which indicates that all alleles supported by more than two reads are reported. To estimate

348 each SSR marker genotype in our samples, 'Koshihikari'- and 'Takanari'-type SNPs were

349 counted from the SSR marker position until one of the counts reached "7" (Supplementary

350 Fig. 4a,b). In the cases where the SNPs around an SSR marker position were too sparse,

351 all the SNPs in $\pm 1.5 \mathrm{Mbp}$ from each SSR marker were counted (Supplementary Fig. 4a,c).

352 Then, each estimated SSR marker was categorized into 'Koshihikari'-type, 'Takanari'-

353 type, or undetermined based on most of its SNP counts (Supplementary Fig. 4a-c). Any samples of the estimated genotypes not perfectly matching the SL1201, SL1229, or markers or to doubtful SNP calls. For the samples with estimated genotypes similar to one of these lines, imputation of estimated SSR marker genotypes was conducted

358 (Supplementary Fig. 4d). Finally, each sample was labeled with the genotype of the most similar SSR marker composition. Thus, 865 samples were confirmed as 'Koshihikari', 
361 'Takanari', or CSSLs in 2016 (Supplementary Fig. 3d).

362 Correlation plot of transcriptomes. Pearson's correlation coefficients of transcriptome

363 data for all pairwise comparisons of the 854 samples from 2015 were calculated as:

$$
\rho_{m, n}=\frac{\sum_{i}\left(y_{i, m}-\bar{y}_{m}\right)\left(y_{i, n}-\bar{y}_{n}\right)}{\sqrt{\sum_{i}\left(y_{i, m}-\bar{y}_{m}\right)^{2}} \sqrt{\sum_{i}\left(y_{i, n}-\bar{y}_{n}\right)^{2}}}
$$

where $\rho_{m, n}$ denotes the Pearson's correlation coefficient between samples $m$ and $n$. Mean

$\log 2$-transformed rpms are denoted as $\bar{y}_{m}$ and $\bar{y}_{n}$, respectively.

with the "image.plot" function in the R package "fields" (version 9.0) $)^{37}$

Meteorological data. Data of average air temperature per $10 \mathrm{~min}$ at the Hirakata Weather

Station $\left(34^{\circ} 48^{\prime} 5^{\prime \prime} \mathrm{N}, 135^{\circ} 33^{\prime} 6^{\prime \prime} \mathrm{E}\right)$ in 2015 for Takatsuki and at Kyotanabe Weather Station

374 function in $\mathrm{R}$ (version 3.4.2) ${ }^{31}$. Data of global solar radiation per minute at the Osaka 
381 the same speed after flowering, the scaled age of sample $m$ was defined as:

$$
a_{m}=\left\{\begin{array}{c}
85 \frac{d_{m}-d_{m}^{S}}{d_{m}^{F}-d_{m}^{S}} \quad\left(d_{m} \leq d_{m}^{F}\right), \\
d_{m}-d_{m}^{F}+85\left(d_{m}>d_{m}^{F}\right),
\end{array}\right.
$$

where $d_{m}$ is the sampling date, $d_{m}^{S}$ is the seeding date, and $d_{m}^{F}$ is the flowering date

384 of each line with genotype and seeding date identical to that of sample $m$.

\section{Calculation of precision weights for each observed normalized read count (log2rpm).}

386 Precision weights were utilized in the training step of FIT (version 0.0 .4$)^{10}$ to deal with over-dispersion of RNA-Seq data ${ }^{10}$. Precision weight for each observed $\log 2 \mathrm{rpm}$ value was calculated based on the residuals from the smoothed time-series as in the voom

$$
\sigma_{i, G}=\sqrt{\frac{1}{\left|M_{G}\right|} \sum_{m \in M_{G}}\left(s_{i, G}\left(t_{m}\right)-y_{i, m}\right)^{2}}
$$


396 where $t_{m}$ denotes sampling time of sample $m . M_{G}$ and $\left|M_{G}\right|$ denote indices of samples

397 whose background genotypes are $G$ and its size, respectively.

398 The mean $\log 2$-transformed read count of gene $i$ in $G$-background genotype was then calculated as:

$$
\bar{c}_{i, G}=\frac{1}{\left|M_{G}\right|} \sum_{\mathrm{m} \in \mathrm{M}_{\mathrm{G}}} y_{i, m}+\frac{1}{|M|} \sum_{m \in M} \log _{2} C_{m}+1-\log _{2} 10^{6},
$$

where $M$ denotes sample indices in both background genotypes.

A LOWESS curve ${ }^{39}$ was then fitted to the square-root of residual standard deviations as

a function of mean log2-transformed read counts, resulting in $l()$, which represents the deviations (Supplementary Fig. 14b).

smoothed log2-transformed read counts of gene $i$ in sample $m$ as:

$$
\hat{c}_{i, m}=s_{i, G_{m}}\left(t_{m}\right)+\log _{2} C_{m}+1-\log _{2} 10^{6}
$$

where $G_{m}$ denotes the background genotype of sample $m$.

410 Finally, precision weight for observed $\log 2 \mathrm{rpm}$ of gene $i$ in sample $m$ was calculated as

411 (Supplementary Fig. 14c):

$$
w_{i, m}=\frac{1}{l\left(\hat{c}_{i, m}\right)^{4}} \text {. }
$$


414 for gene expression dynamics in 'Koshihikari' and 'Takanari' was developed with the R

415 package "FIT" (version 0.0 .4$)^{10}$. In this section, we describe how "FIT" developed a

416 prediction model.

417 There are several strategies for the integrative analysis of the transcriptome and

418 meteorological data to investigate environmental responses in field conditions ${ }^{40-44}$. In the

419 FIT package, we employed the statistical modeling approach for a comprehensive

420 relationship among these data and for understanding the effects of age and circadian clock

421 of plants. We used the transcriptome data in combination with the meteorological data,

422 namely air temperature and global solar radiation, which were measured at a

423 meteorological station near the field from where the samples were acquired. Because

424 there was no remarkable disease or pest damage during cultivation and no difference in

425 fertilization among samples, the model did not include these effects.

426 The $\log 2 \mathrm{rpm}$ of the normalized expression for gene $i$ of sample $m$ was described by a

427 simple linear model:

$$
y_{i, m}=\alpha_{i}+\boldsymbol{x}_{i, m}^{T} \boldsymbol{\beta}_{i}+\epsilon_{i, m}
$$

429 where $\alpha_{i}, \boldsymbol{x}_{i, m}$, and $\boldsymbol{\beta}_{i}$ are a constant term, a vector of eight explanatory variables, and

430 regression coefficients, respectively. The third term $\varepsilon_{i, m}$ is the independently and

431 identically distributed noise drawn from a Gaussian distribution. The explanatory 
432 variables were plant's scaled age, circadian clock, optional variable, response to

433 environmental stimuli, and the interactions between scaled age and circadian clock and

434 between scaled age and response to environmental stimuli. The optional variable was not used in this study. The plant's scaled age corresponded to the scaled number of days after transplanting. See section "Conversion of age to scaled age" for the definition of scaled age. The values of the scaled age were normalized to mean 0 and variance 1 . The circadian clock, with the peak at an arbitrary time, was represented by two variables corresponding to its cosine and sine components:

$$
\begin{aligned}
& \cos \left(\frac{2 \pi t_{m}}{24}\right), \\
& \sin \left(\frac{2 \pi t_{m}}{24}\right),
\end{aligned}
$$

441 where $t_{m}$ is the time at which sample $m$ was obtained. Similarly, the interaction between scaled age and circadian clock of a plant was represented by two variables. exhibit more complex responses. The complexity of fluctuations in gene expression is mainly explained by the nonlinearity of the response to the environmental stimuli, which

446 is the cumulative sum of the nonlinearly transformed environmental stimuli for a given period. Such cumulative sum of temperature, for example, is designated as cumulative temperature and it is widely used for the prediction of reproduction timing and vegetation 
449 in ecology and of harvest timing in agriculture ${ }^{45,46}$. By applying this to gene expression,

450 the environmental response of gene $i$ in sample $m$ can be described as:

$\hat{e}_{i, m}=\sum_{\tau=t_{m}-p_{i}}^{t_{m}} g_{i}(\tau) \max \left(0, \tanh \left( \pm \exp \left(\gamma_{i}^{(f)}\right)\left(e_{\tau}-\theta_{i}^{(f)}\right)\right)\right) \times \sqrt{\exp \left(-2 \gamma_{i}^{(f)}\right)+1}$

where $p_{i}$ is the period during which gene expression was affected by an environmental

stimulus, $e_{\tau}$ is the value of a meteorological parameter at time $\tau$ normalized to mean 0 and

variance $1, \theta_{i}^{(f)}$ is the response threshold to the stimulus, and $\gamma_{i}^{(f)}$ is the parameter that

consisted of air temperature and global solar radiation. Of these parameters, the one

yielding the highest likelihood was selected for each gene in the optimization procedure.

458 In the limit $\gamma_{i}^{(f)} \rightarrow \infty$, a gene responds to an environmental stimulus in a dose-

exceeds the threshold (Supplementary Fig. 15). Conversely, as this parameter approaches

Fig. 15). The sign of the value in the hyperbolic function determines whether the gene

465 likelihood. The last term $\sqrt{\exp \left(-2 \gamma_{i}^{(f)}\right)+1}$ ensures that the scale of the response is 466 largely independent of this parameter. The function $g_{i}(\tau)$ is a gate function that explains 
467 the time-of-day-specific environmental responses. A gate function is defined as:

$$
g_{i}(\tau)=n_{i}\left(\tanh \left(\exp \left(\gamma_{i}^{(g)}\right)\left(\cos \left(2 \pi\left(\tau-\psi_{i}\right)\right) / 24-\theta_{i}^{(g)}\right)\right)\right)
$$

469 where $\gamma_{i}^{(g)}$ controls the shape of the gate as $\gamma_{i}^{(f)}$ in the environmental response, $\theta_{i}^{(g)}$

473 the gate is a constant value in the limit $\gamma_{i}^{(g)} \rightarrow \infty$, that is, the gate is always open 474 (Supplementary Fig. 15). minimized is thus defined as:

$$
\begin{aligned}
& L(\boldsymbol{\beta})=\sum_{m \in M}\left(\hat{y}_{i, m}-y_{i, m}\right)^{2} \\
& +\lambda\left(\sum_{k \in I} \zeta_{k}\left|\beta_{k}\right|+\zeta_{c} \sqrt{\beta_{c o s}^{2}+\beta_{\text {sin }}^{2}}+\zeta_{d c} \sqrt{\beta_{d c o s}^{2}+\beta_{d s i n}^{2}}\right)
\end{aligned}
$$

where $\lambda$ and $\zeta$ are the regularization parameter and the adaptive weights, respectively. 
484 The values of the adaptive weights are decided as in the adaptive lasso ${ }^{48}$ or the adaptive

485 group lasso ${ }^{49}$. Further, the adaptive weights were used to absorb differences in the degrees

486 of freedom by multiplying the covariates of the environmental response and interaction

487 between the environmental response and scaled age by seven, as seven free parameters

488 were considered. We selected the value of $\lambda$ as the largest value for which the cross

489 validation (CV) error was smaller than the sum of the minimum CV error and its standard

error.

The values of the parameters related to environmental responses, which are

$492 p_{i}, \theta_{i}^{(f)}, \gamma_{i}^{(f)}$, and the sign of the value in the hyperbolic function in Eq. (12), and $493 \theta_{i}^{(g)}, \gamma_{i}^{(g)}$, and $\psi_{i}$ in Eq. (13), are selected by the Nelder-Mead algorithm ${ }^{50}$. Because the 494 likelihood function is complex and has multiple local maxima, a grid search was performed before optimization. In each step of the optimization by the Nelder-Mead algorithm, we performed an ordinary least square regression instead of group lasso (Eq. (14)) to reduce the computational cost. 'Koshihikari' and 'Takanari'-prediction models were developed based on timeseries RNA-Seq data of the 'Koshihikari'-background CSSLs and 'Koshihikari' and of the 'Takanari'-background CSSLs and 'Takanari' in 2015, respectively (Supplementary Fig. 2b). Values of the expressed genes (a matrix of log2rpm for each sample), sample 
502 attributes (a matrix of sampling year, month, day, hour, and min and scaled age for each

503 sample), weather data (a matrix of air temperature and global solar radiation at every

504 minute in 2015), and precision weights for the matrix of log2rpm of each sample

505 (Supplementary Fig. 16) were prepared. Details on the parameters for 'FIT' are listed in

506 https://github.com/naganolab/Rice_edQTL-analysis_and_edQTL-based-

507 prediction/analysis.R. As a result, the functions $f_{i, G}()$ were yielded for gene $i$. A log2rpm

508 of gene $i$ of sample $m$ was predicted as (Supplementary Fig. 16):

$$
\hat{y}_{m}=f_{i, G_{m}}\left(t_{m}, a_{m}, \boldsymbol{e}\right)
$$

510 where $\boldsymbol{e}$ denotes meteorological data (air temperature and global solar radiation) at every

511 minute.

512 Detection of genes with expression dynamics polymorphism. To increase detection

513 power in multiple tests, we focused on genes with expression dynamics polymorphism.

514 Euclidean distances of gene $i$ between 'Koshihikari'- and 'Takanari'-prediction models

$\left(d_{i}\right)$ were calculated as:

$$
d_{i}=\sqrt{\sum_{m \in M}\left(f_{i, \mathrm{~K}}\left(t_{m}, a_{m}, \boldsymbol{e}\right)-f_{i, \mathrm{~T}}\left(t_{m}, a_{m}, \boldsymbol{e}\right)\right)^{2}} .
$$

517 As a result, 3696 genes with $d_{i}>40$ were defined as genes with expression dynamics polymorphism (Supplementary Fig. 3e).

519 Detection of edQTL. According to background genotypes, the sum of residual errors 
520 between the observed $\log 2 \mathrm{rpm}$ and predicted $\log 2 \mathrm{rpm}$ of gene $i$ in the 'Koshihikari'- or

521 'Takanari'-models $\left(E_{i}\right)$ were calculated as (Supplementary Fig. 3a):

$$
\begin{gathered}
E_{i, m}=\left|\hat{y}_{i, m}-y_{i, m}\right|, \\
E_{i}=\sum_{m \in M} E_{i, m},
\end{gathered}
$$

524 where $E_{\text {im }}$ denotes the residual error between the observed $\log 2 \mathrm{rpm}$ and predicted

$\log 2 \mathrm{rpm}$ of gene $i$ in sample $m$ on the assumption that the 'Koshihikari'- or 'Takanari'-

526 type gene expression dynamics shown by sample $m$ is determined based on background

527 genotype $G_{m}$. Similarly, on the assumption that edQTL for gene $i$ exist around SSR marker

$528 g$, the sum of residual errors between the observed log2rpm and predicted log2rpm of gene $i$ in the 'Koshihikari'- or 'Takanari'-models $\tilde{E}_{i, g}$ were calculated as

530 (Supplementary Fig. 3a):

$$
\begin{gathered}
\tilde{E}_{i, m, g}=\left|f_{i, Q_{g, m}}\left(t_{m}, a_{m}, \boldsymbol{e}\right)-y_{i, m}\right| \\
\tilde{E}_{i, g}=\sum_{m \in M} \tilde{E}_{i, m, g}
\end{gathered}
$$

533 where $\tilde{E}_{i, m, g}$ denotes the residual error between the observed log2rpm and predicted

$534 \log 2 \mathrm{rpm}$ of gene $i$ in sample $m$ and $Q_{g, m}$ denotes genotype of SSR marker $g$ in sample

$535 m$. Thus, $f_{i, Q_{g m}}\left(t_{m}, a_{m}, \boldsymbol{e}\right)$ denotes predicted log2rpm in sample $m$ assuming the 536 existence of edQTL for gene $i$ around SSR marker $g$. The improvement in residual errors assuming that edQTL for gene $i$ exists around $g$ was therefore calculated as 
538 (Supplementary Fig. 3a,b):

$$
I_{i, g}=E_{i, m}-\tilde{E}_{i, m, g}
$$

To evaluate the statistical significance of the improvement in the sum of residual

541 errors, 1000 permutations of samples with 'Koshihikari'- and 'Takanari' genetic

542 background were performed (Supplementary Fig. 6e). For each permutation, the

543 improvement in residual errors on the assumption that edQTL for gene $i$ exist around SSR

544 marker $g$ was calculated (Supplementary Fig. 6b). Then, we generated the null

545 distribution of improvements in residual errors by fitting log-normal distribution to

546 obtained improvements using the fitdist function in the R package "fitdistrplus"51, with

547 the fitting method of quantile matching of $70 \%$ and $1 \%$ (Supplementary Fig. 6c). For

548 almost all genes with expression dynamics polymorphism, the 99.9 percentiles of the

549 fitted log-normal distributions were higher than (2863/3696 genes) or almost the same as

550 those of the distribution obtained by the permutations (Supplementary Fig. 6d), indicating

551 that the $p$-value obtained from log-normal distribution was conservative. Thus, the $p$ -

552 value for the improvement in the sum of residual errors for gene $i$ on the assumption that

553 an edQTL affecting gene expression dynamics of gene $i$ exists around SSR marker $g$ was

554 obtained based on the null distribution. 
556 the Benjamini-Hochberg method ${ }^{52}$ using the p.adjust function in R. Finally, we defined

557 the markers with the smallest adjusted $p$-value $(<0.05)$ in each peak for each gene as the

558 edQTL affecting each gene expression dynamics.

$$
g_{i, m}=\left\{\begin{array}{l}
\tilde{G}_{m},\left(\exists g \in \mathcal{G}_{i}, Q_{g, m} \neq G_{m}\right), \\
G_{m}, \text { otherwise, }
\end{array}\right.
$$

where $\widetilde{\mathrm{G}}_{m}$ denotes the opposite genotype to that of the genetic background in sample $m$,

$\log 2 \mathrm{rpm}$ of gene $i$ in sample $m$ was calculated as:

$$
\hat{y}_{i, m}^{\mathrm{ED}}=f_{i, g_{i, m}}\left(t_{m}, a_{m}, \boldsymbol{e}\right)
$$

Statistical test for the accuracy of gene expression dynamics prediction in BILs. The calculated as:

$$
E_{G}=\sum_{i \in I^{\mathrm{ED}}, m \in M^{\mathrm{B}}}\left|f_{i, G}\left(t_{m}, a_{m}, \boldsymbol{e}\right)-y_{i, m}\right|,
$$




$$
E^{\mathrm{ED}}=\sum_{i \in I^{\mathrm{ED}}, m \in M^{\mathrm{B}}}\left|\hat{y}_{i, m}^{\mathrm{ED}}-y_{i, m}\right|,
$$

575 where $M^{\mathrm{B}}$ denotes the indices of BIL samples and $I^{\mathrm{ED}}$ denotes the genes affected by 576 the edQTL.

578 errors, 10,000 permutations were performed for the markers in each BIL genome. In each

579 permutation, the sums of prediction errors of all genes affected by the edQTL for the BILs

580 were calculated with the edQTL model. Then, the value corresponding to percentile 0.1

581 of the sums of prediction errors was calculated.

582

583 Code availability. The scripts used in this study are available from

584 https://github.com/naganolab/Rice_edQTL-analysis_and_edQTL-based-prediction.

586 Data availability. All datasets generated and/or used in this study are available from

PRJDB7234. 
589 1. Poorter, H. et al. Pampered inside, pestered outside? Differences and similarities between plants growing in controlled conditions and in the field. New Phytol. 212, 838-855 (2016). plants: the missing heritability is in the field. Genome Biol. 12, 232 (2011). causes of complex disease. Nat. Rev. Genet. 11, 446-450 (2010). temperature response functions. Nat. Plants 3, 17102 (2017). differences in rice evolution result in two contrasting types of differentially expressed genes. BMC Genomics 1099, 16 (2015).

601 6. Wang, J. et al. An expression quantitative trait loci-guided co-expression analysis for constructing regulatory network using a rice recombinant inbred line population. J. Exp. Bot. 65, 1069-1079 (2014).

$6047 . \quad$ Jansen, R. C. \& Nap, J. P. Genetical genomics: The added value from 
8. Wang, J. et al. A global analysis of QTLs for expression variations in rice shoots at the early seedling stage. Plant J. 63, 1063-1074 (2010). tool for transcriptome dynamics under fluctuating field conditions.

11. Nagano, A. J. et al. Deciphering and prediction of transcriptome dynamics under fluctuating field conditions. Cell 151, 1358-1369 (2012).

12. Matsuzaki, J., Kawahara, Y. \& Izawa, T. Punctual transcriptional regulation by the rice circadian clock under fluctuating field conditions. Plant Cell 27, 633-648 (2015). resistance using a set of reciprocal chromosome segment substitution lines in rice. Sci. Rep. 30572, 6 (2016). 
621 14. Takai, T. et al. Genetic mechanisms underlying yield potential in the rice high-

622

623

624

625

626

627

628

629

630

631

632

633

634

635

636

yielding cultivar Takanari, based on reciprocal chromosome segment substitution lines. BMC Plant Biol. 295, 14 (2014).

15. Adachi, S. et al. The mesophyll anatomy enhancing CO2diffusion is a key trait for improving rice photosynthesis. J. Exp. Bot. 64, 1061-1072 (2013).

16. Adachi, S. et al. Genetic architecture of leaf photosynthesis in rice revealed by different types of reciprocal mapping populations. J. Exp. Bot. (2019).

17. Sakamoto, T. An Overview of Gibberellin Metabolism Enzyme Genes and Their Related Mutants in Rice. Plant Physiol. 134, 1642-1653 (2004).

18. Sultan, S. E. Phenotypic plasticity and plant adaptation. Acta Bot. Neerl 44, 363 383 (1995).

19. Fournier-Level, A. et al. Predicting the evolutionary dynamics of seasonal adaptation to novel climates in Arabidopsis thaliana. Proc. Natl. Acad. Sci. U. S. A. 113, E2812-21 (2016).

20. Skelly, D. A., Ronald, J. \& Akey, J. M. Inherited Variation in Gene Expression. Annu. Rev. Genomics Hum. Genet. 10, 313-332 (2009). 
637 21. Kaur, J., Rahat, B., Thakur, S. \& Kaur, J. Trends in Precision Medicine. Prog.

22. Kremling, K. A. G. G. et al. Dysregulation of expression correlates with rareallele burden and fitness loss in maize. Nature 555, 520-523 (2018).

23. Horinouchi, T. et al. Prediction of cross-resistance and collateral sensitivity by gene expression profiles and genomic mutations. Sci. Rep. 14009, 7 (2017). plant viruses in natural environments by using RNA-Seq. Plant Virol. Protoc. (2012). Illumina sequence data. Bioinformatics 30, 2114-2120 (2014). 
genome using next generation sequence and optical map data. Rice 6, 4 (2013).

654

655

656

657

658

659

660

661

662

663

664

665

666

667

28. Sakai, H. et al. Rice annotation project database (RAP-DB): an integrative and interactive database for rice genomics. Plant Cell Physiol. 54, 1-11 (2018).

29. Li, B. \& Dewey, C. N. RSEM: accurate transcript quantification from RNA-Seq data with or without a reference genome. BMC Bioinformatics 12, 323 (2011).

30. Langmead, B., Trapnell, C., Pop, M. \& Salzberg, S. L. Ultrafast and memoryefficient alignment of short DNA sequences to the human genome. Genome Biol. 10, R25 (2009).

31. R Core Team. R: A language and environment for statistical computing. $\mathrm{R}$ Foundation for Statis-tical Computing, Vienna, Austria. (2017).

32. Kamitani, M., Nagano, A. J., Honjo, M. N. \& Kudoh, H. RNA-Seq reveals virusvirus and virus-plant interactions in nature. FEMS Microbiol. Ecol. 92, 1-11 (2016).

33. Langmead, B. \& Salzberg, S. L. Fast gapped-read alignment with Bowtie 2. Nat. Methods 9, 357-359 (2012). 
Bioinformatics 25, 2078-2079 (2009).

670

671

672

673

674

675

676

677

678

679

680

681

682

683

684
35. Danecek, P. et al. The variant call format and VCFtools. Bioinformatics 27, 2156-2158 (2011).

36. Knaus, B. J. \& Grünwald, N. J. vcfr: a package to manipulate and visualize variant call format data in R. Mol. Ecol. Resour. 17, 44-53 (2017).

37. Nychka, D., Furrer, R., Paige, J. \& Sain, S. fields: Tools for spatial data. (2017). doi:10.5065/D6W957CT

38. Law, C. W., Chen, Y., Shi, W. \& Smyth, G. K. voom: precision weights unlock linear model analysis tools for RNA-seq read counts. Genome Biol. 15, R29 (2014).

39. Cleveland, W. S. LOWESS: A Program for Smoothing Scatterplots by Robust Locally Weighted Regression. Am. Stat. 35, 54 (1981).

40. Wilkins, O. et al. EGRINs (Environmental gene regulatory influence networks) in rice that function in the response to water deficit, high temperature, and agricultural environments. Plant Cell 28, 2365-2384 (2016).

41. Cronn, R. et al. Transcription through the eye of a needle: Daily and annual 
685

686

687

688

689

690

691

692

693

694

695

696

697

698

699

cyclic gene expression variation in Douglas-fir needles. BMC Genomics 18, (2017).

42. Richards, C. L., Rosas, U., Banta, J., Bhambhra, N. \& Purugganan, M. D.

Genome-wide patterns of Arabidopsis gene expression in nature. PLoS Genet. 8, (2012).

43. Izawa, T. et al. Os- GIGANTEA Confers Robust Diurnal Rhythms on the Global Transcriptome of Rice in the Field. Plant Cell 23, 1741-1755 (2011).

44. Sjödin, A., Wissel, K., Bylesjö, M., Trygg, J. \& Jansson, S. Global expression profiling in leaves of free-growing aspen. BMC Plant Biol. 8, (2008).

45. Kira, T. On the altitudinal arrangement of climatic zones in Japan-a contribution to the rational land utilization in cool highlands. Kanti-Nogaku 2, 143-173 (1948).

46. Schwartz, M. Phenology : an integrative environmental science. (Springer, 2014).

47. Yuan, M., Yuan, M. \& Lin, Y. Model selection and estimation in regression with grouped variables. J. R. Stat. Soc. Ser. B 68, 49--67 (2006). 
701 48. Zou, H. The adaptive lasso and its oracle properties. J. Am. Stat. Assoc. 101,

49. Wang, H. \& Leng, C. A note on adaptive group lasso. Comput. Stat. Data Anal. 52, 5277-5286 (2008).

50. Nelder, J. A. \& Mead, R. A Simplex Method for Function Minimization.

Comput. J. 7, 308-313 (1965).

707

51. Delignette-Muller, M. L. \& Dutang, C. fitdistrplus : An R Package for Fitting Distributions. J. Stat. Softw. 64, 1-34 (2015). 
713 Supplementary Information is available in the online version of the paper.

715 Acknowledgments. We thank the field work support team for help at field sites, Fumie

716 Kobayashi for assistance with laboratory experiments, and Yasuhiro Sato for valuable

717 discussions. We thank Toshio Yamamoto (National Agriculture and Food Research

718 Organization) for providing seeds of the CSSLs and BILs between 'Koshihikari' and

719 'Takanari'. This work was supported by the JST CREST JPMJCR15O2 and JSPS

720 KAKENHI Grants JP16H06171 and JP16H01473 to A.J.N.

722 Author contributions. M.K. analyzed the data. R.L.S., H.S., S.O. and S. A. performed

723 field work. M.K., A.T., A.D., Y.H., and Y.K. conducted RNA-Seq experiments. K.I.

724 developed the FIT library. A.J.N. designed the study. M.K. and A.J.N. wrote the

725 manuscript with input from all co-authors.

727 Declaration of interests. The authors declare no competing interests. 
a
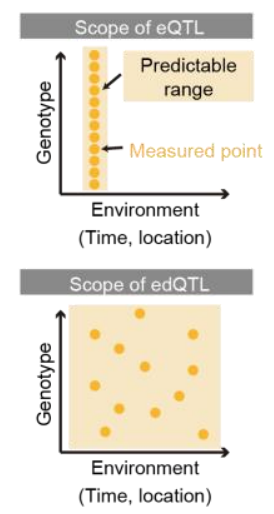

c

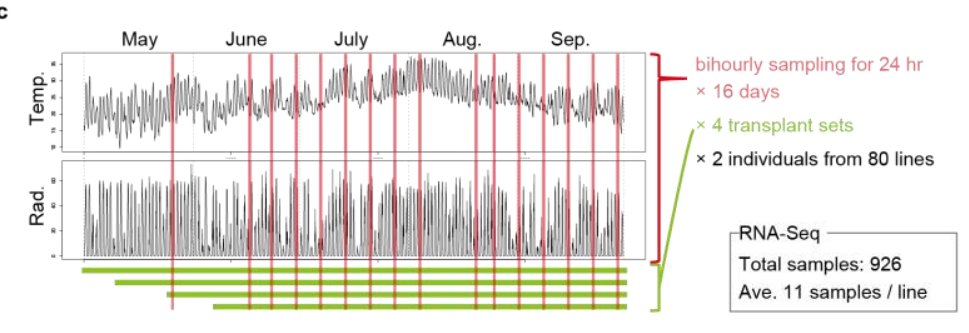

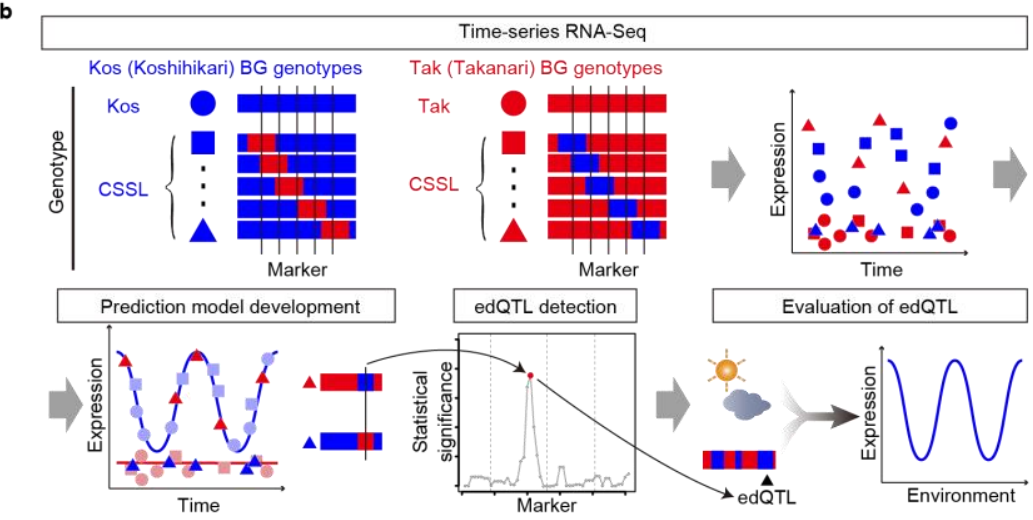

d

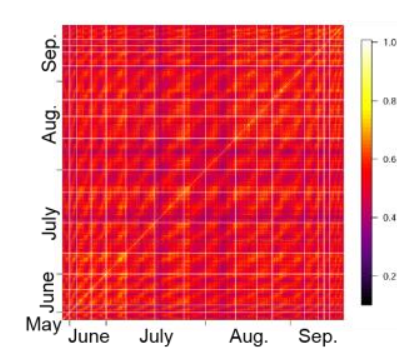

729

730 Figure 1 | Concept and workflow of expression dynamics quantitative trait loci

731 (edQTL) detection. a, Conceptual differences in the scopes of expression quantitative

trait loci (eQTL) and edQTL. b, Workflow of edQTL detection and its evaluation using edQTL detection. The left panels show plots of meteorological data [air temperature (Temp., ${ }^{\circ} \mathrm{C}$ ) and global solar radiation (Rad., $\left.\left.\mathrm{kJ} \mathrm{m}^{-2} \mathrm{~min}^{-1}\right)\right]$ in Takatsuki from May to

September in 2015. Vertical red lines represent the sampling time points. d, Pearson's 
bioRxiv preprint doi: https://doi.org/10.1101/451609; this version posted January 31, 2020. The copyright holder for this preprint (which was not certified by peer review) is the author/funder. All rights reserved. No reuse allowed without permission.

739 bihourly sampling set. 

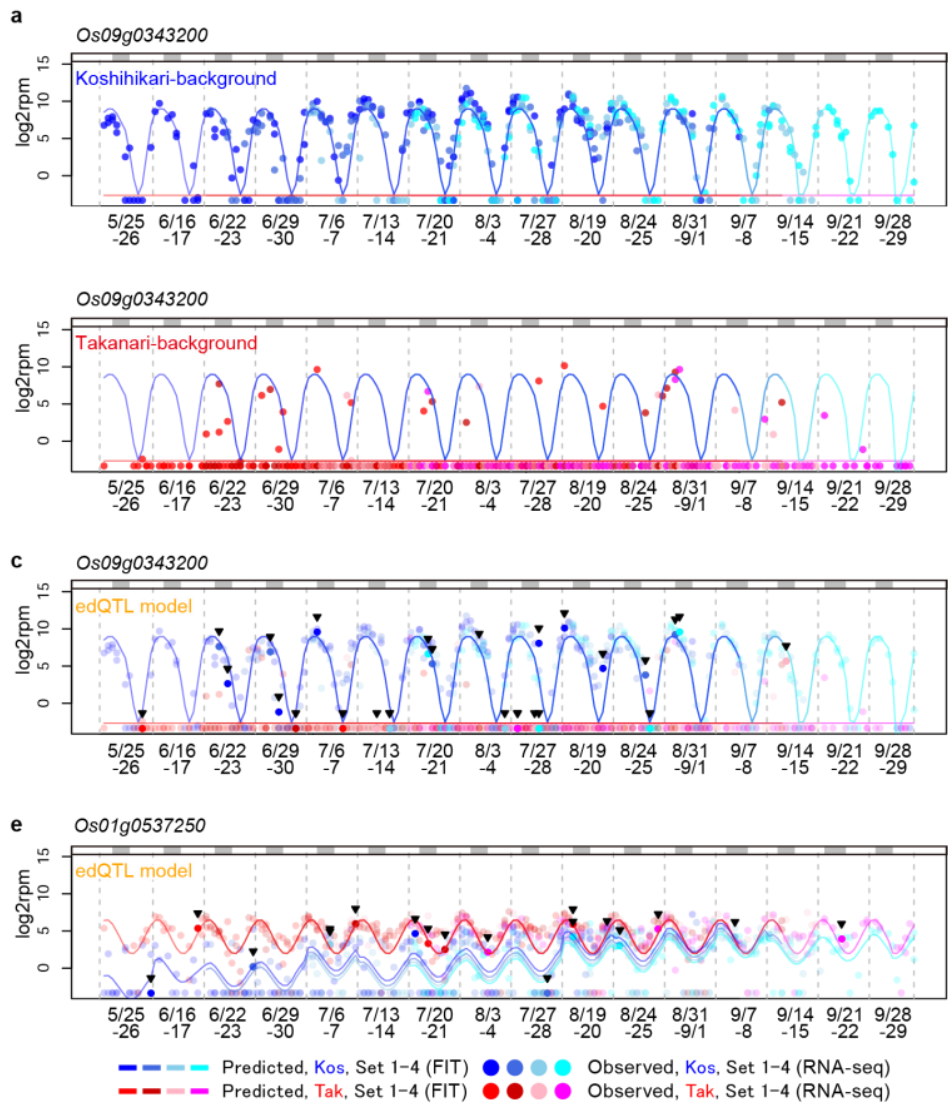
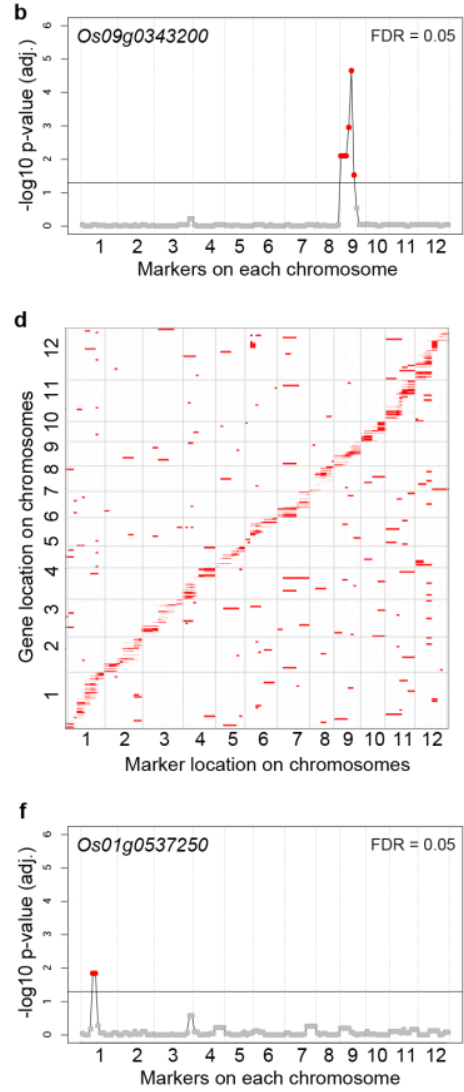
'FIT' based on scaled age and environmental information (time, air temperature, and global solar radiation).Blue and red/pink lines indicate predicted expression levels in 'Koshihikari' and 'Takanari' in transplant sets 1, 2, 3, and 4, respectively. Blue and red/pink points indicate the expression level obtained by RNA-sequencing for samples in transplant sets 1, 2, 3, and 4 of individuals with 'Koshihikari'-background genotype (BG) 
751 respectively. In (c,e), strong colored points emphasized by the arrowheads indicate

752 samples harboring edQTL different from their BGs, and light-colored points indicate the

753 samples harboring edQTL identical to their BGs. The upper gray bar indicates dark

754 periods (global solar radiation $<0.3 \mathrm{~kJ} \mathrm{~m}^{-2} \mathrm{~min}^{-1}$ ). b,f, edQTL regulating Os09g0343200

755 (b) and $0 s 01 g 0537250$ (f). d, Position of the 1675 edQTL are shown as red bars (false

756 discovery rate $=0.05)$. $\mathrm{X}$-axis and $\mathrm{Y}$-axis represent the positions of markers with edQTL

757 and the positions of genes influenced by edQTL, respectively. 
a

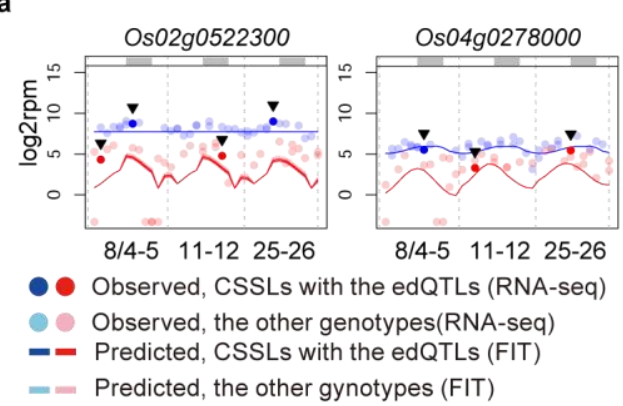

b Background VS edQTL

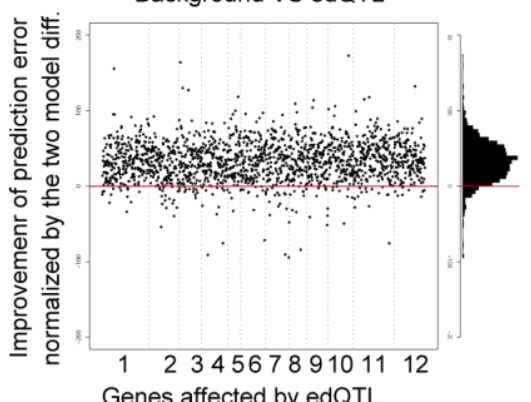

Genes affected by edQTL

d Permutation
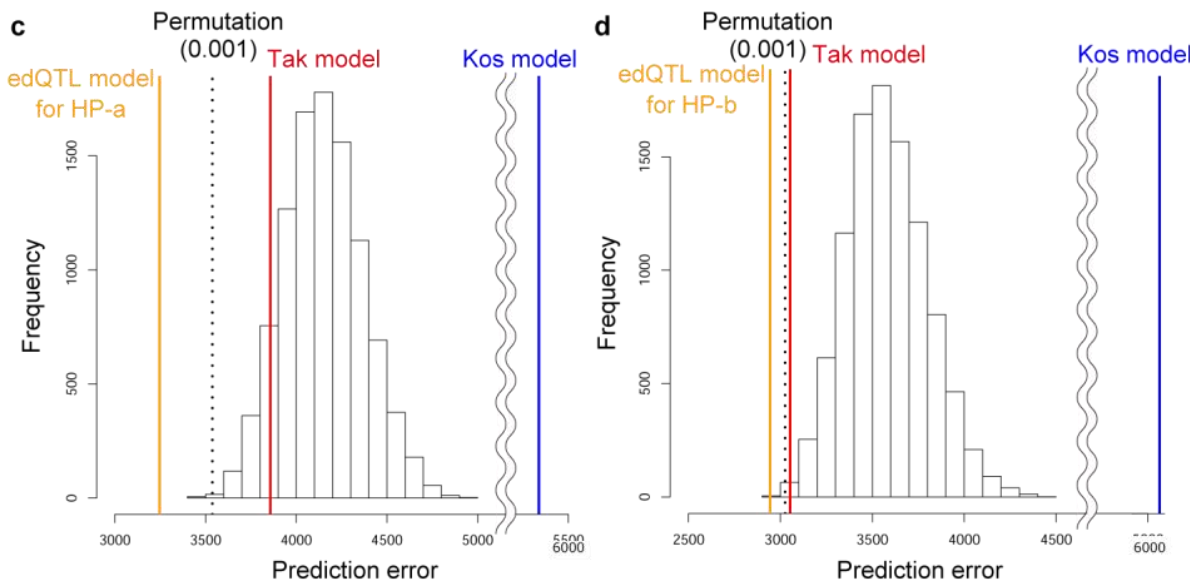

e

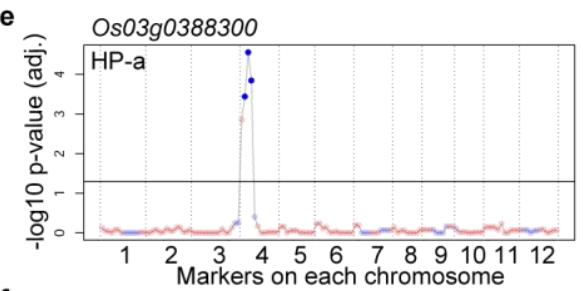

f

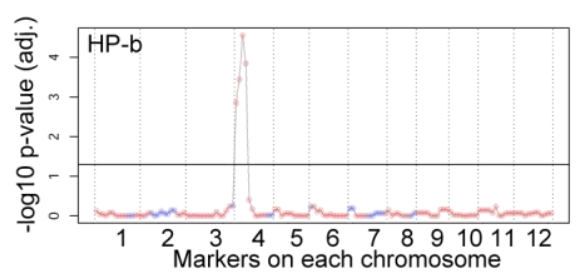

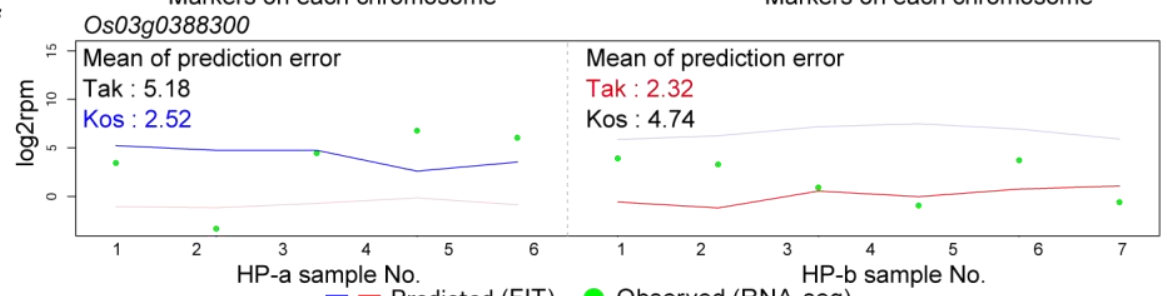

759 Figure 3 | Expression dynamics quantitative trait loci (edQTL)-based prediction of

Examples of prediction of expression dynamics in Kizugawa in 2016 based on 
763 environmental information and edQTL in transplant set 1. Points in intense colors

764 emphasized by arrowheads indicate samples harboring edQTL different from their

765 background genotypes and light colors indicate samples harboring edQTL identical to

766 their background genotypes. The upper gray bars indicate dark periods (global solar

767 radiation $\left.<0.3 \mathrm{~kJ} \mathrm{~m}^{-2} \mathrm{~min}^{-1}\right)$. $\mathbf{b}$, Effects of edQTL over the prediction of gene expression

768 dynamics in Kizugawa in 2016. Genes influenced by edQTL are in order of position on

769 the chromosomes along the horizontal axis. c,d, Prediction accuracy of edQTL model for

770 HP-a (c) and HP-b (d). The blue, red, and orange vertical lines indicate the sums of

771 prediction errors based on 'Koshihikari', 'Takanari', and edQTL models. The histogram

772 shows the distribution of the sums of prediction errors based on the edQTL model in

77310,000 permutations of markers in HP-a or HP-b genomes. The dashed vertical line

774 indicates the $0.1 \%$ percentile of the distribution. e, edQTL for $O s 03 g 0388300$ and

775 genotypes of HP-a and HP-b. Dark blue points indicate significant 'Koshihikari'-type

markers. f, Prediction of Os03g0388300 expression in HP-a and HP-b. Intense color lines 


\section{Supplemental Tables}

780 Table S1 SSR marker composition in the rice lines used in the present study

781 "Position" indicates the position of each SSR marker on the chromosomes in the

782 IRGSP1.0 reference genome. A and B indicate 'Koshihikari' - and 'Takanari'-type alleles,

783 respectively.

784

785

Table S2 Sample attributes used in this study

786 "TransPlantSet" indicates the transplant sets in each year. Details of seeding and

787 transplant dates in each set are described in the methods. "Year", "month", "day", and

788 "hour" columns indicate the sampling date. The "LineName" column indicates the

789 predicted genotypes based on SNPs detected in each RNA-Seq. The "field" column

790 indicates the location of the fields.

Table S3 Oryza sativa genes and their expressions

793 The "Expression" column indicates whether the genes are "expressed genes" or not. "Yes"

794 indicates that "expressed genes" were detected in more than $20 \%$ of all samples. The

795 "Polymorphism" column indicates whether the genes show expression dynamics

796 polymorphism between 'Koshihikari' and 'Takanari' or not. "Yes" indicates that the 
797 Euclidian distances of predicted expression dynamics between 'Koshihikari' and

798 'Takanari' were more than 40. The "edQTL", "cis.edQTL", and "trans.edQTL" columns

799 indicate whether the genes have the designated edQTL or not. 